\title{
Adaptive Sparse Tiling for Sparse Matrix Multiplication
}

\author{
Changwan Hong \\ The Ohio State University \\ Columbus, OH, USA \\ hong.589@osu.edu
}

\author{
Aravind Sukumaran-Rajam \\ The Ohio State University \\ Columbus, OH, USA \\ sukumaranrajam.1@osu.edu
}

\author{
Israt Nisa \\ The Ohio State University \\ Columbus, OH, USA \\ nisa.1@osu.edu
}

\author{
Kunal Singh \\ The Ohio State University \\ Columbus, OH, USA \\ singh.980@osu.edu
}

\author{
P. Sadayappan \\ The Ohio State University \\ Columbus, $\mathrm{OH}$, USA \\ sadayappan.1@osu.edu
}

\begin{abstract}
Tiling is a key technique for data locality optimization and is widely used in high-performance implementations of dense matrix-matrix multiplication for multicore/manycore CPUs and GPUs. However, the irregular and matrix-dependent data access pattern of sparse matrix multiplication makes it challenging to use tiling to enhance data reuse. In this paper, we devise an adaptive tiling strategy and apply it to enhance the performance of two primitives: SpMM (product of sparse matrix and dense matrix) and SDDMM (sampled dense-dense matrix multiplication). In contrast to studies that have resorted to non-standard sparse-matrix representations to enhance performance, we use the standard Compressed Sparse Row (CSR) representation, within which intra-row reordering is performed to enable adaptive tiling. Experimental evaluation using an extensive set of matrices from the Sparse Suite collection demonstrates significant performance improvement over currently available state-ofthe-art alternatives.
\end{abstract}

CCS Concepts • Computing methodologies $\rightarrow$ Shared memory algorithms; • Computer systems organization $\rightarrow$ Single instruction, multiple data;

Keywords Sparse Matrix-matrix Multiplication, Sampled Dense-dense Matrix Multiplication, SpMM, SDDMM, GPU, multicore/manycore, Tiling

\footnotetext{
Permission to make digital or hard copies of all or part of this work for personal or classroom use is granted without fee provided that copies are not made or distributed for profit or commercial advantage and that copies bear this notice and the full citation on the first page. Copyrights for components of this work owned by others than ACM must be honored. Abstracting with credit is permitted. To copy otherwise, or republish, to post on servers or to redistribute to lists, requires prior specific permission and/or a fee. Request permissions from permissions@acm.org.

PPoPP '19, February 16-20, 2019, Washington, DC, USA

(C) 2019 Association for Computing Machinery.

ACM ISBN 978-1-4503-6225-2/19/02 . .\$15.00

https://doi.org/10.1145/3293883.3295712
}

\section{ACM Reference Format:}

Changwan Hong, Aravind Sukumaran-Rajam, Israt Nisa, Kunal Singh, and P. Sadayappan. 2019. Adaptive Sparse Tiling for Sparse Matrix Multiplication. In 24th ACM SIGPLAN Symposium on Principles and Practice of Parallel Programming (PPoPP '19), February 16-20, 2019, Washington, DC, USA. ACM, New York, NY, USA, 15 pages. https://doi.org/10.1145/3293883.3295712

\section{Introduction}

Tiling is a key technique for effective exploitation of data reuse and is used in all high-performance implementations of dense linear algebra computations, convolutional neural networks, stencil computations, etc. While tiling for such regular computations is well understood and is heavily utilized in high-performance implementations on multicore/manycore CPUs and GPUs, the effective use of tiling for sparse matrix multiplication poses challenges.

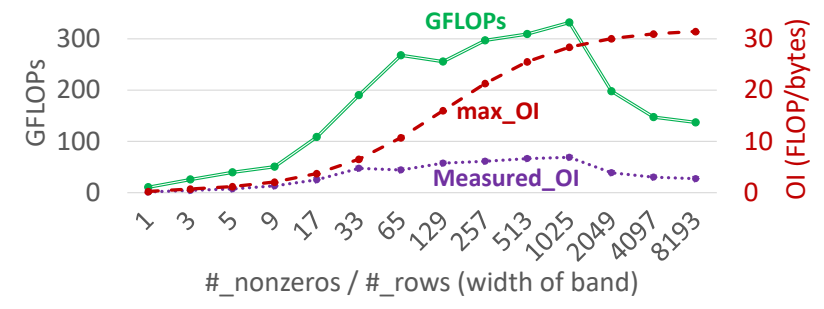

Figure 1. OI and GFLOPs with respect to matrices having different bands

In order to motivate the need for data locality optimization via tiling for sparse-matrix dense-matrix multiplication $(\mathrm{SpMM})^{1}$, we present some experimental data on an Intel Xeon Phi processor (KNL, Knights Landing) using the $m k l \_s c s r m m$ routine for SpMM in the Intel MKL library. A number of banded matrices $(\{S \mid S[x][y] \neq 0 \Longleftrightarrow(0 \leq$ $x<\#$ rows, $\max (0, y-b) \leq y<\min (\# \operatorname{cols}, y+b))\}$, where

\footnotetext{
${ }^{1}$ We use SpMM to denote the product of a sparse matrix with a dense matrix, to be distinguished from sparse matrix-matrix multiplication (SpGEMM), where two sparse matrices are multiplied.
} 
$\mathrm{b}$ is the half band-size) of size $16 K \times 16 K$ with different band-sizes were used as the sparse matrix argument for the MKL SpMM routine. Fig. 1 presents the performance trend (GFLOPs) as the band-size is varied. Performance improves up to a band-size of 1025 and drops beyond that. The figure also plots the measured operational intensity (OI), the ratio of floating-point operations to the number of bytes of data moved to/from main memory. It may be seen that measured OI increases up to a band-size of 1025 and then drops for larger band sizes. Thus, the performance drop is correlated with increased data movement per operation. As we explain in greater detail later in Sec. 3, this is in contrast to the potential maximum OI, which increases with band-size.

With dense matrix-matrix multiplication, uniform tiling is the norm, where all tiles (except boundary tiles) have the same number of operations and same data footprint. However, with SpMM the number of non-zero elements will vary significantly across different uniform-sized tiles due to the very non-uniform distribution of non-zero elements across the $2 \mathrm{D}$ index space of a sparse matrix. As we explain in detail later in the paper, whether tiled execution can achieve higher performance than untiled execution for a $2 \mathrm{D}$ region of a sparse matrix depends on the sparsity structure in that region., In this paper, we develop an Adaptive Sparse Tiling (ASpT) approach to tiling two variants of sparse matrix multiplication: SpMM (Sparse-dense Matrix Multiplication) and SDDMM (Sampled Dense Dense Matrix Multiplication). A key idea is that the average number of non-zeros per "active" row/column segment (i.e., at least one nonzero) within a $2 \mathrm{D}$ block plays a significant role in determining whether tiled or untiled execution is preferable for a 2D block. The sparse matrix is partitioned into panels of rows, with the active columns within each row-panel being either grouped into 2D tiles for tiled execution, or relegated to untiled execution because its active column density is inadequate. In contrast to other prior efforts that have used customized data representations in order to improve the performance of sparse matrix operations, we achieve the hybrid tiled/untiled execution by using the standard (unordered) Compressed Sparse Row (CSR) representation of sparse matrices: the nonzero elements in column segments that are to be processed in untiled mode are reordered to be contiguously located at the end in the unordered CSR format.

We demonstrate the effectiveness of the proposed modeldriven approach to hybrid-tiled execution of sparse matrix computations by developing implementations for SpMM and SDDMM kernels on GPUs and multicore/manycore processors (Intel Xeon, and Intel Xeon Phi KNL). On all platforms, the significant performance improvement is achieved over available state-of-the-art alternatives - Intel's MKL and Nvidia's cuSPARSE libraries for SpMM, and the MIT TACO compiler and BIDMach for SDDMM.

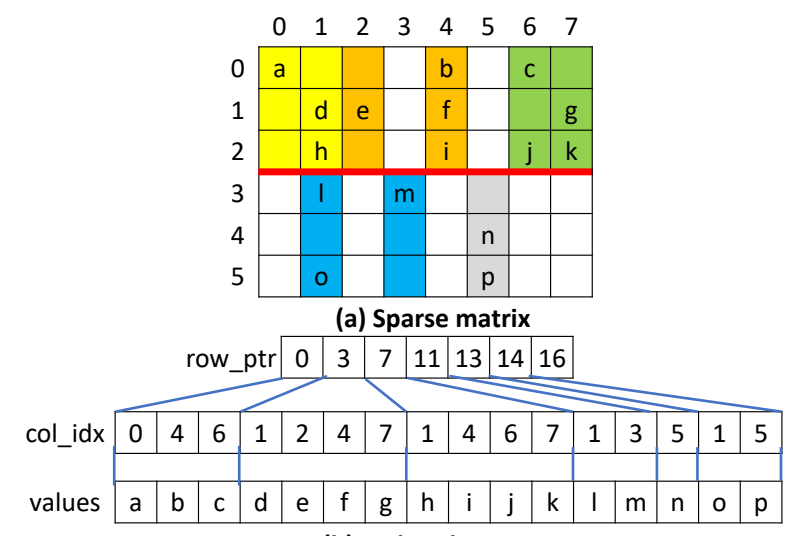

(b) Ordered CSR

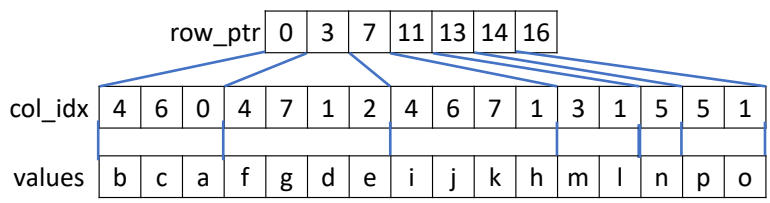

(c) Unordered CSR

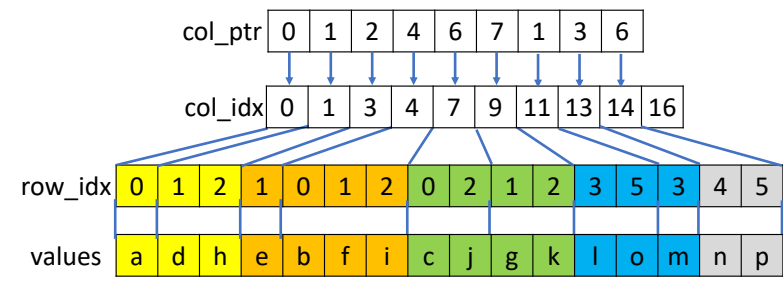

(d) DCSC

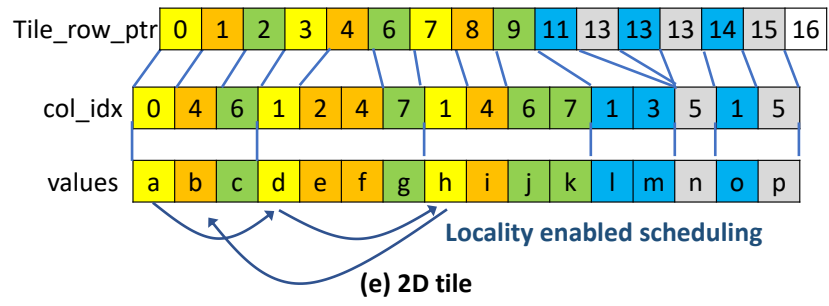

Figure 2. Various data representations for a sparse matrix

\section{Background and Related Work}

\subsection{Standard Sparse Matrix Representation}

The CSR representation is one of the most widely used data structures for representing sparse matrices [35, 48]. As shown in Fig. 2 (b,c), the CSR structure is composed of three arrays: row_ptr, col_idx, and values. The value of row_ptr[i] contains the index of the first element of row $i$. values[] holds the actual numerical values of the nonzero elements, and col_idx[] holds the corresponding column indices. As shown in Fig. 2 (b,c), non-zeros within each row are placed contiguously in col_idx[] and values[]. CSR has two variants, ordered CSR and unordered CSR [19]. In ordered CSR, the column indices within a row are sorted, whereas in unordered CSR the column indices may not be kept in sorted order. 
Fig. 2 (c) illustrates unordered CSR and Fig. 2 (b) represents the corresponding ordered CSR version.

Double-Compressed Sparse Column (or Row) DCSC (DSCR) [6] is an alternate format, used for ultra-sparse matrices where many rows (columns) may be completely empty. Fig. 2 (d) shows a DCSC representation, where a sparse matrix is partitioned into row panels. Four arrays are maintained: col_ptr[], col_idx[], row_idx[], and values[]. col_idx[] contains the column index and col_ptr[] points to the first element of the corresponding column segment. row_idx[] and values[] store the row indices and the actual non-zero values, respectively. Fig. 2 (d) shows the DCSC representation.

Sparse matrices can also be represented using 2D-tiles, as shown in Fig. 2 (e). Like DCSC, the sparse matrix is partitioned into a set of row panels. Each row panel is further divided into 2D tiles. tile_row_ptr[] is used to track the start point of each row within a 2D tile. The col_idx[] and values[] hold the column indices and actual non-zero values, respectively.

\subsection{SpMM and SDDMM}

In SpMM, a sparse matrix $S$ is multiplied by a dense matrix $D$ to form a dense output matrix $O$. Fig. 3 (left) shows a conceptual view of SpMM. Alg. 1 shows sequential SpMM using a CSR representation. SpMM is widely used in many applications such as Locally Optimal Block Preconditioned Conjugate Gradient (LOBPCG) for finding eigenvalues of a matrix [3], Convolutional Neural Networks (CNNs) [16], and graph centrality calculations [33]. SpMM is also one of the core GraphBLAS primitives [7].

In SDDMM two dense matrices D1 and D2 are multiplied and the result matrix is then scaled by an input sparse matrix $S$ (Hadamard product). Fig. 3 (right) shows a conceptual view of SDDMM. Alg. 2 shows sequential SDDMM using a CSR representation. The SDDMM primitive can be used for efficient implementation of many applications such as Gamma Poisson (GaP) [39], Sparse Factor Analysis (SFA) [10], and Alternating Least Squares (ALS) [21].

Both SpMM and SDDMM traverse the rows of S (the outer loop). In SpMM, each element in the i-th row (with column index k) of the input sparse matrix $S$ is used to scale the k-th row of $D 1[k][:]$ and the partial products are accumulated to form the $\mathrm{i}$-th row of the output matrix $O[i][:]$. In SDDMM, the dot product of the j-th row of D1 (i.e., D1[j][:]) and i-th row of $D 2$ (i.e., D2[i][:]) is computed at nonzero position (i,j) of the sparse matrix $S$ and then scaled with $S(i, j)$ to form $\mathrm{O}(\mathrm{i}, \mathrm{j})$.

Several recent research efforts have been directed toward the development of efficient Sparse Matrix-Vector Multiplication (SpMV) [14, 23, 24, 27, 28, 32, 34-38, 40, 46, 49]. However, very few efforts have focused on SpMM and SDDMM.

In typical applications where SpMM or SDDMM are used, these operations are repeated many times using the same
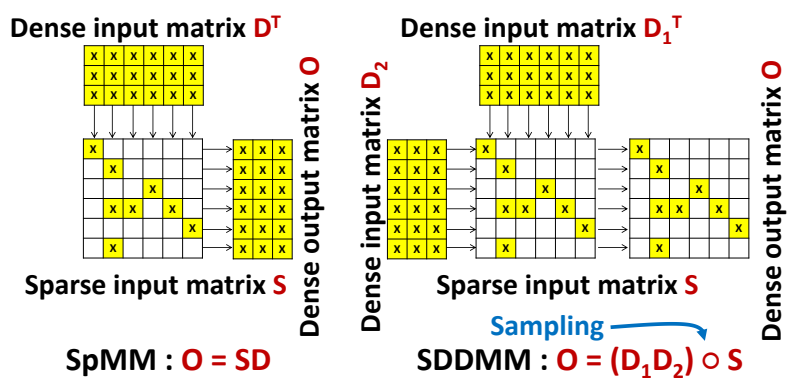

Figure 3. Conceptual view of SpMM and SDDMM

sparse matrix (the values may change, but the sparsity structure does not change). For instance, SpMM is useful in the Generalized Minimum Residual (GMRES) [4] method, where several hundred iterations are required. This usage pattern allows a one-time light pre-processing of the sparse matrix to enhance performance, and the cost of this pre-processing is amortized across the iterations. As explained later, our approach requires a one-time reordering of sparse matrix elements to enhance data locality and reuse.
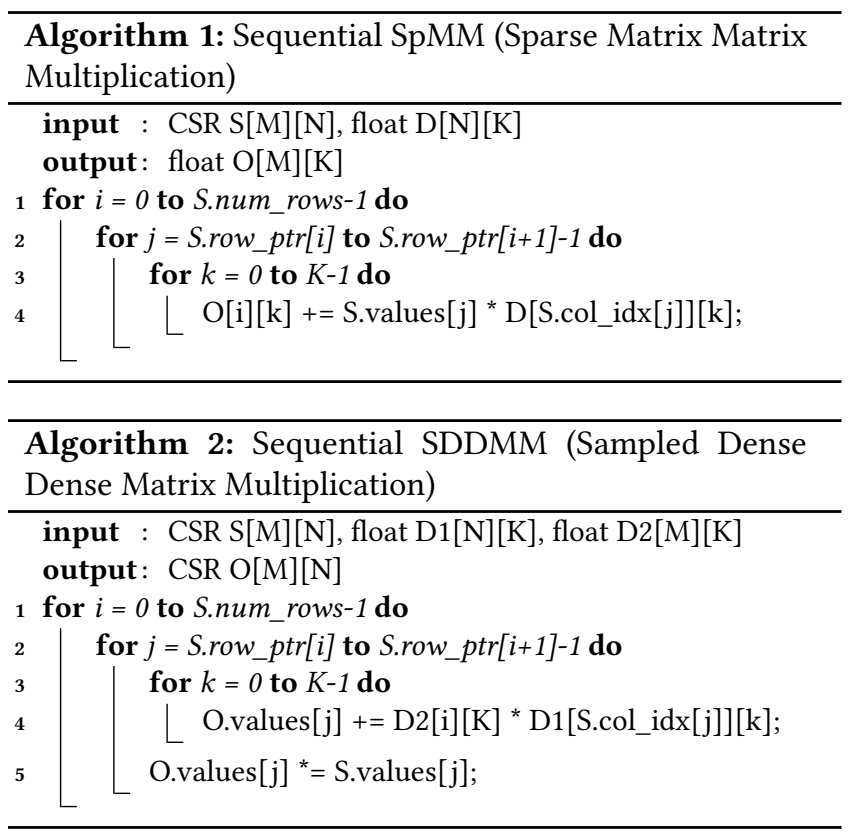

\subsection{Related Work}

Efforts to optimize SpMM and SDDMM may be grouped into two categories: using standard representation (CSR) or non-standard customized sparse matrix representation.

Intel's MKL [43] is a widely used library for multi/many cores. MKL includes optimized kernels for many sparse matrix computations, including SpMM, SpMV and sparsematrix sparse-matrix multiplication (SpGEMM).

TACO [20] is a recently developed library using compiler techniques to generate kernels for sparse tensor algebra 
operation, including SpMM and SDDMM. Generated kernels are already optimized, and OpenMP parallel pragma is used for parallelization.

cuSPARSE [1] provided by Nvidia also supports SpMM. It offers two different modes depending on access patterns of dense matrices (i.e., row or column major order).

BIDMach [11] is a library for large-scale machine learning, and includes several efficient kernels for machine learning algorithms such as Non-negative Matrix Factorization (NMF), Support Vector Machines (SVM). It includes an implementation of SDDMM.

Recently, Yang et al. [48] applied row-splitting [5] and merge-based [27] algorithms to SpMM to efficiently hide global memory latency. Based on the pattern of the sparse matrix, one of two algorithms is applied.

Several efforts have sought to improve SpMM performance by defining new representations for sparse matrices. Variants of ELLPACK have been used to improve performance, e.g., ELLPACK-R in FastSpMM [30], and SELL-P in MAGMA [3].

OSKI [41] uses register blocking to enhance data reuse in registers/L1-cache which improves the SpMV performance. When the nonzero elements are highly clustered, register blocking can reduce the data footprint of the sparse matrix.

Compressed Sparse Blocks (CSB) [2] is another sparse matrix storage format which exploits register blocking. The sparse matrix is partitioned and stored as small rectangular blocks. In CSB, register blocking also reduces the overhead of transposed SpMM $\left(O=A^{T} B\right)$. SpMM implementation with CSB data representation has been demonstrated to achieve high performance when both SpMM and transposed SpMM $\left(O=A^{T} B\right)$ are simultaneously required [2]. Register blocking also plays a pivotal role in many sparse matrix formats for both CPUs [8, 9, 45] and GPUs [47].

We recently developed an SpMM implementation for GPUs based on a hybrid sparse matrix format called RS-SpMM that enabled significant performance improvement over alternative SpMM implementations [17]. However, a disadvantage of the approach is that a customized non-standard data structure is used for representing the sparse matrix, making it incompatible with existing code bases and libraries. Applications often use many library functions, which are based on the CSR representation. Iterative applications that make repeated use of SpMM interleaved with other sparse matrix operations may incur a high overhead in a repeated conversion from standard CSR to the non-standard representation $[35,48]$.

Reordering of sparse matrices has been widely explored in many other contexts. Yzelman et al. [50] show that reordering by recursive hypergraph-based sparse matrix partition can enhance cache locality, and thus performance. Oliker et al. [29] demonstrate that the performance of conjugate gradient (CG) and incomplete factorization (ILU) preconditioning can be improved by several reordering techniques such as METIS graph partitioning [18], that enhance locality.
While the above reordering strategies improve performance, they suffer from significant pre-processing overhead. GOrder [44] and ReCALL [22] try to reduce the preprocessing overhead using greedy strategy for graph algorithms. The key idea is to number/index the vertices such that vertices with many common neighbors are assigned indices which are close to each other to improve data locality.

In this paper, we seek to improve the SpMM/SDDMM performance without the use of any non-standard sparse matrix representations. A significant benefit of using an unordered CSR format rather than an arbitrary new data format is the compatibility with existing code and libraries. Another benefit is the reduced storage space requirement. With non-standard representations, it may be necessary to keep an additional copy of the sparse-matrix in a standard format such as CSR for use with other library functions. By using a standard representation, this space overhead can be avoided.

There are two significant differences between the reordering technique used in ASpT and existing works. First, the pre-processing overhead is significantly lower (milliseconds) than reordering schemes like GOrder [44] and ReCALL [22], which take tens of seconds for SuiteSparse datasets with large numbers of non-zeros [13]. Second, the original indexing of vertices is preserved, which eliminates overheads for re-indexing.

\section{Overview of ASpT}

This subsection provides an overview of ASpT (Adaptive Sparse-matrix Tiling), a strategy for tiled execution of sparse matrix multiplication using an unordered Compressed Sparse Row (CSR) representation.

We first elaborate on the observed performance trend shown earlier in Fig. 1. Let us consider the execution of the CSR SpMM algorithm (Alg. 1). The outer (i) loop traverses the rows of the sparse matrix $S$; the middle $(j)$ loop accesses the nonzero elements in row $w_{i}$ of $\mathrm{S}$, and the inner $(\mathrm{k})$ loop updates $r w_{i}$ of the output array $O$ by scaling appropriate rows of the input dense matrix $D$ by the values of the nonzero elements of $S$ in row $_{i}$. The total number of non-zero elements for an $N \times N$ banded matrix with band-size $B$ is approximately $N B$ and so the total number of floating point operations for SpMM product with a dense matrix of size $N \times K$ is 2 NBK. The total data footprint for the computation (sum of sizes of all arrays) for single-precision (4 bytes per word for the two dense matrices; 8 bytes per nonzero in the sparse matrix, for column index and value; 4 bytes per row pointer in CSR) is $4 \mathrm{NK}+4 \mathrm{NK}+8 \mathrm{NB}+4 \mathrm{~N}$, or approximately $8 \mathrm{~N}(\mathrm{~K}+\mathrm{B})$. The maximum possible operational intensity $(\mathrm{OI})$, corresponding to complete reuse of data elements in cache/registers is thus $\frac{2 N B K}{8 N(K+B)}=\frac{1}{\frac{4}{B}+\frac{4}{K}}$. Therefore, as the band-size increases, max_OI also increases. The actually achieved (measured) OI first increases as $B$ increases, but then decreases due to 
limited L2 cache capacity. The Intel Xeon Phi KNL system has a 1 Mbyte L2 cache shared by two cores. The inner $(\mathrm{k})$ loop in Alg. 1 traverses $K$ distinct elements, and the middle (j) loop traverses $B$ iterations, resulting in access of a total of $B K$ elements of $D$. With a banded matrix, the set of column indices for adjacent rows almost completely overlap (except for two elements at the ends of the band), resulting in almost complete reuse of the data from $D$ if sufficient cache capacity is available. For $\mathrm{K}=128$, setting $4^{*} 128^{*} \mathrm{~B}=512 \mathrm{~K}$ gives $\mathrm{B}=1024$, consistent with the experimental data that shows a drop in performance when B is raised from 1025 to 2049.

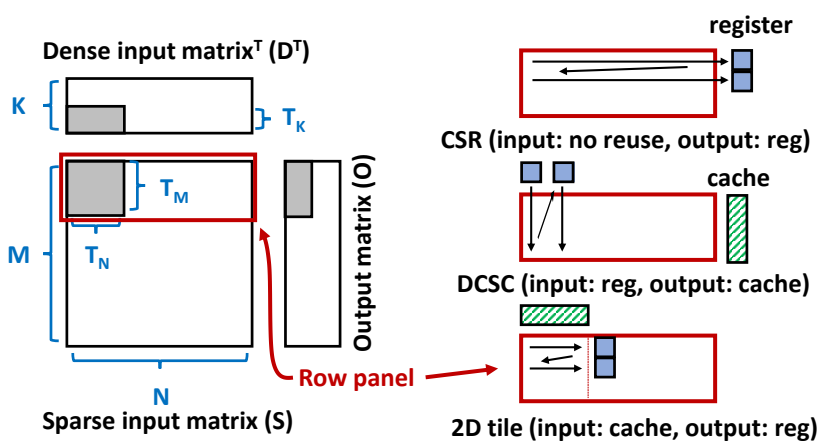

Figure 4. Data Reuse with three different data representations

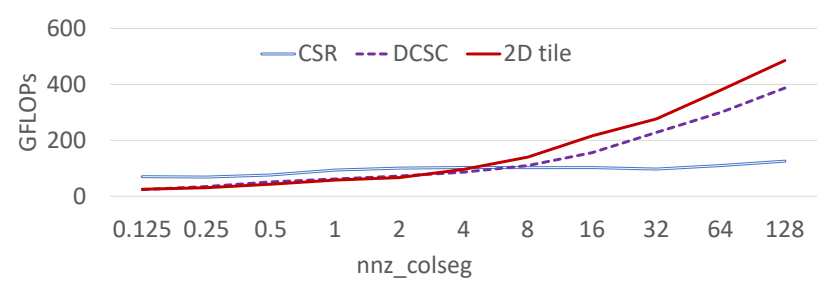

Figure 5. Performance of CSR, DCSC, 2D tile for different synthetic matrices

Thus it can be seen that data reuse for the input dense matrix $D$ may suffer significantly if too many other rows are accessed before a row is again referenced (when the next nonzero in the corresponding column of $\mathrm{S}$ is accessed). The extent of achieved reuse of $\mathrm{D}$ is thus very dependent on the sparsity structure of S. In the extreme case, for Alg. 1, no reuse at all may be achieved for $D$, while full reuse is achieved for $S$ and $O$. This is illustrated in Fig. 4 - the element $O[i][j]$ in Alg. 1 being placed in a register because of its repeated access in the innermost loop.

In order to achieve better reuse for elements of $D$, the order of access of the elements of $S$ must be changed. For a banded sparse matrix, full reuse of $D$ can be achieved by accessing $S$ column-wise. But full column-wise access will result in loss of reuse for $O$. By performing column-wise access within row-panels of $S$, it is feasible to still achieve full reuse for $O$ in cache, as well as some reuse for $D$. This corresponds to the use of DCSC data representations, with the access pattern shown in Fig. 4. While this DCSC scheme may be superior in terms of minimization of data movement to/from memory, its access pattern may be detrimental to ILP (Instruction Level Parallelism) due to the very small average number of nonzeros within the active columns in a row-panel. Further, the number of register loads/stores increases with this scheme since each operation requires a load-modify-store from/to registers. A third alternative is to use $2 \mathrm{D}$ tiling, as shown in Fig. 4, where access is row-wise within a tile, allowing better register-level reuse for the accumulated results.

The trade-offs between these three alternatives depend on the sparsity structure of the matrix and are very difficult to model analytically due to the complex interplay between the impact of the reduction of the volume of data access from memory and increase in stall cycles due to reduced ILP. Therefore we used micro-benchmarks based on synthetic random matrices to understand the performance trends for the three alternative schemes shown in Fig. 4. Fig. 5 shows the performance (single precision) for different synthetic sparse matrices on an Intel Xeon Phi KNL. The non-zeros in the synthetic matrices are randomly distributed with different sparsity, and nnz_colseg (the average number of elements in a column segment in DCSC) is computed as $\frac{T_{M} \times n n z}{M \times N}$ where $T_{M}$ is the recommended row segment size for DCSC, and $\mathrm{nnz}$ is the number of non-zeros in the sparse matrix with $M=128 \mathrm{~K}, N=4 \mathrm{~K}, K=128$. To fully exploit the L2 cache on KNL, the row panel size is chosen as 512 and 256, for DCSC and $2 \mathrm{D}$ tile, respectively (the row panel size is halved for 2D tile since the cache is used for both dense input $D$ and dense output $O$ matrices).

With CSR, the non-zero elements of the sparse matrix in a row are accessed one by one and multiplied by the corresponding elements in $D$. The partial results are accumulated in registers and written out to memory at the end of each row. The $O$ elements get full reuse in registers. However, the reuse of $D$ elements is very dependent on the sparsity structure of $S$ and for large sparse matrices the reuse for these elements can be very low. In other words, with the CSR representation, it is difficult to exploit locality for $D$. This performance impact is shown in Fig. 5

Performance can be improved by exploiting reuse of $D$ elements. when the number of elements in a column segment ( $n n z_{-}$colseg) is high. DCSC targets the improvement of reuse of $D$. In DCSC (Fig. 4), the sparse matrix is partitioned into a set of row panels, each of which has $T_{M}$ contiguous rows. The size of a row panel $\left(T_{M}\right)$ is chosen such that the corresponding $O$ elements can fit in the L1/L2 cache (or shared memory in case of GPUs), i.e., $T_{M} \times T_{K} \leq$ cache size. Each non-empty column-segment of the row panel is processed sequentially. The $D$ elements corresponding to the column are brought into registers and the partial results are accumulated in the cache. Thus, within a row panel, the $D$ elements get full reuse 
from registers and the $O$ elements get full reuse from the cache. In DCSC, the reuse for $D$ is increased, but the reuse of $O$ elements is from the cache as opposed to registers in CSR (register accumulations are faster). Hence, as shown in Fig. 5, the performance with a DCSC representation increases with $n n z$ colseg. When $n n z$ colseg is low, the standard CSR representation outperforms DCSC.

$2 \mathrm{D}$ tiling can be used to achieve good reuse of $D$ and $O$ elements. In 2D tiling (in Fig. 4), the sparse matrix is partitioned into a set of row panels which are further subdivided into a set of $2 \mathrm{D}$ tiles such that each tile has $T_{M}$ rows and $T_{N}$ columns of the sparse matrix. The elements within a 2D tile are represented in CSR format (other formats can also be used). In this scheme, the $D$ elements are loaded to cache and the partial accumulations in each row of the $2 \mathrm{D}$ tile are done in registers (similar to CSR). However, as shown in Fig. 5, when $n n z$ colseg is low, the performance of the 2D tiling scheme is lower than CSR.

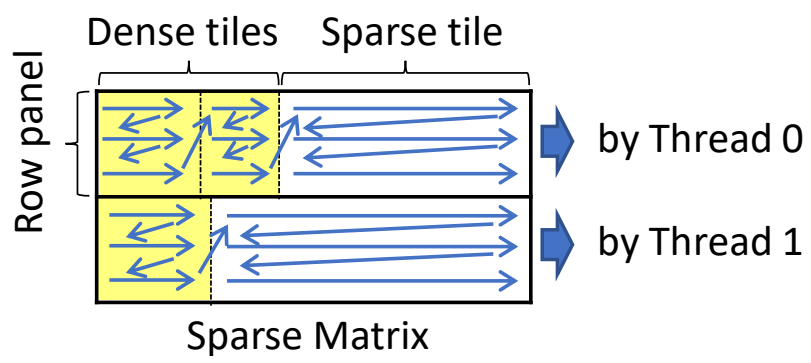

Figure 6. SpMM with ASpT on many cores

The ASpT scheme is based on the observation that when columns in a 2D tile have sufficiently high $n n z$ _colseg, 2D tiling achieves the best performance. When $n n z$ _colseg is low, row-wise access with the standard CSR algorithm is best. Our empirical evaluation with synthetic benchmarks did not reveal scenarios where DCSC performance is the best. Therefore, we use a combination of row-wise CSR access and 2D-tiled execution. Fig. 6 shows the high-level idea behind the Adaptive Sparse Tiling (ASpT) approach that we describe in detail in the next section. The sparse matrix is first divided into row-panels, where the row-panel size is determined by cache/scratchpad capacity constraints. Within each rowpanel, column segments are classified as sufficiently dense or not (the threshold is dependent on the target system and is determined from the cross-over point between CSR and 2D-Tile performance with the micro-benchmarking using synthetic matrices (e.g., Fig. 5). The columns within a rowpanel are then reordered so that columns over the threshold are placed in 2D tiles (the horizontal sizing of the 2D tiles is explained in the next section), while all columns below the threshold are placed at the right end of the row panel in a large group targeted for untiled row-wise CSR execution.

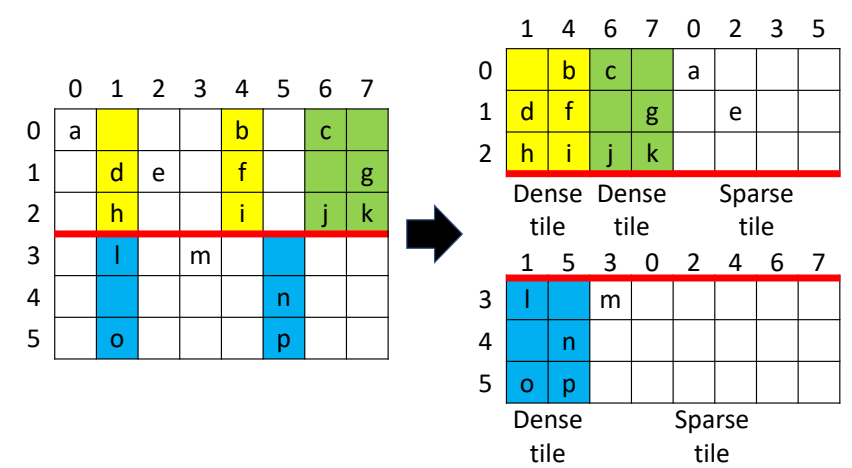

(a) Reordering each row panel of sparse matrix

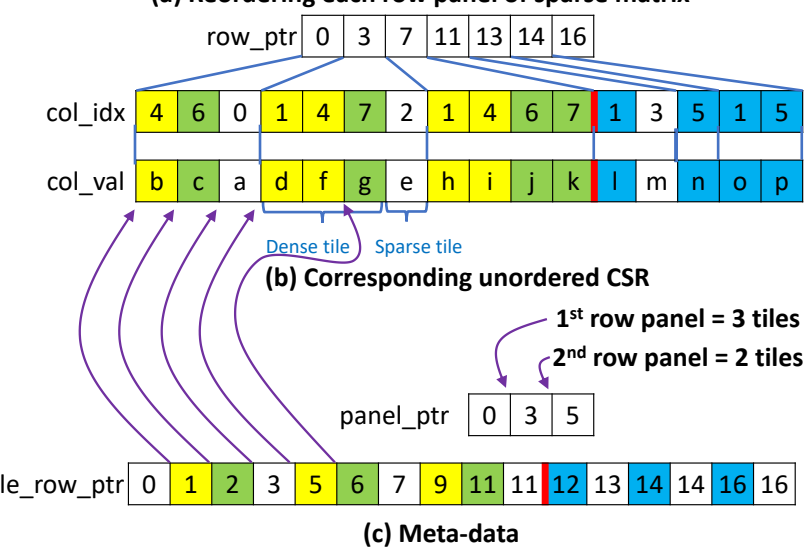

Figure 7. Splitting sparse matrix into heavily clustered rowsegments and remainder

\section{SpMM with ASpT}

\subsection{Data Representation}

Our SpMM scheme uses the unordered CSR representation with additional metadata, as depicted in Fig. 7. Fig. 7 (a) and (b) show the conceptual view of the sparse matrix and the corresponding unordered CSR representation, respectively (the corresponding ordered CSR representation is seen in Fig. 2 (b)). In Fig. 7 (a), the entire matrix is split into two rowpanels, where each row-panel contains a set of contiguous rows. A column segment within a row panel is classified as heavy if it has at least two non-zeros. Each row panel of the sparse matrix is reordered as seen in Fig. 7 (b). All the heavy columns in a row panel are placed before the light columns. Each reordered row panel can thus be viewed as two segments, where the first segment contains a set of heavy columns and the second segment consists of light columns. The first segment (heavy) is further subdivided into 2D tiles, while the entire second segment is viewed as a single 2D tile. The width of the $2 \mathrm{D}$ tiles in the heavy segments of row panels is selected such that the corresponding elements of $D$ fit in the cache (or shared memory). We note that our approach only performs reordering of non-zeros and not re-numbering (i.e., column indexes of the non-zero elements remain unchanged). 
Additional metadata in 'tile_row_ptr' keeps track of the start and end pointers of each tile (Fig. 7 (c)) within each row. For example, consider the first row of the reordered matrix. The first tile begins at position ' 0 '. Hence, tile_row_ptr[0] is ' 0 '. The first element corresponding to the second tile begins at position ' 1 '; hence tile_row_ptr[1] is ' 1 ', and so on. The number of 2D tiles in each row panel is encoded in panel_ptr[i]. For a given row panel, the number of $2 \mathrm{D}$ tiles can be obtained by subtracting panel_ptr[i] from panel_ptr $[i+1]$.

\subsection{SpMM on Multi/many-cores}

Listing 1. SpMM with ASpT on multi cores

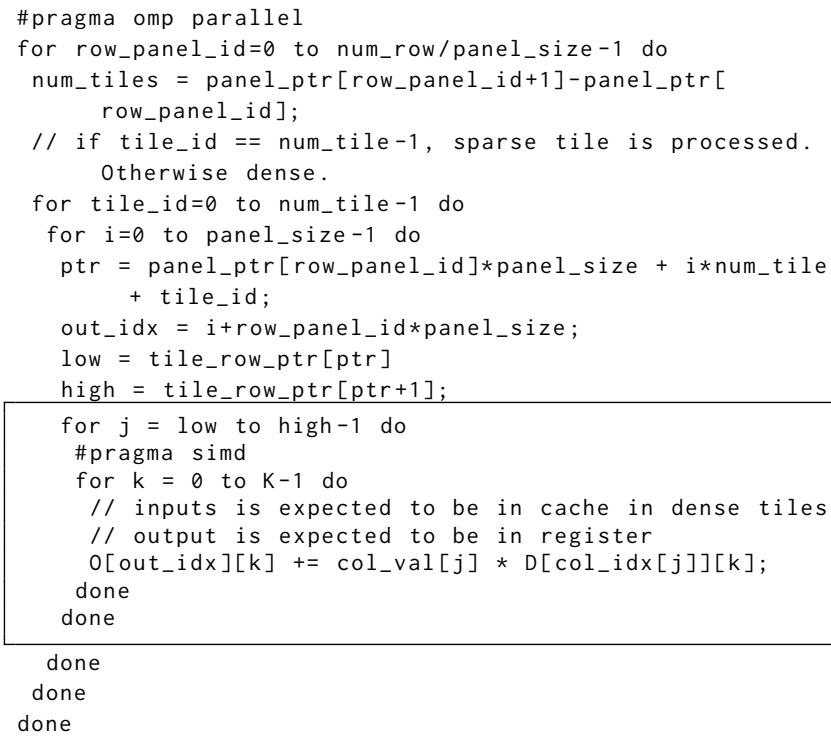

Listing 1 shows the ApST SpMM algorithm specialized for multi/many-core processors. The row panels of the sparse matrix are distributed among threads (Line 2-21). As mentioned in the previous sub-section, the entire row-panel is split to a set of heavy tiles (tile_id < num_tile -1$)$ and a single sparse tile (tile_id $==$ num_tile -1$)$. Both the heavy tiles and sparse tiles are processed by the same kernel; however, we expect most of the $D$ accesses in heavy tiles to be served by the L2 cache and from memory for the sparse tiles. The tiles within a row panel are processed sequentially (Line 5). The elements of each row within a 2D tile are identified by using tile_row_ptr[0] and panel_ptr[i+1] (line 7 to 10). The non-zero elements in each row of the $2 \mathrm{D}$ tile are processed sequentially (line 11-18). In order to increase Instruction Level Parallelism (ILP), vectorization is done along the $\mathrm{K}$ dimension. This also helps to achieve good cache line utilization.

\subsection{SpMM on GPUs}

Although we can use the same high-level tiling idea for GPUs, the GPU implementation should take advantage of the GPU architecture in order to achieve high performance. The main difference between GPUs and multi/many-core processors is that GPUs have many more registers per thread. It also has an explicitly managed scratchpad memory called shared memory. However, cache capacity per thread in GPUs is quite small $\left(\frac{\text { \#ache size }}{\# \text { of threads on SM or SMs }}\right)$.

\subsubsection{Utilization of Shared Memory and Registers}

Contrary to multi/many-cores, where only a few threads access L1/L2 cache simultaneously, a huge number of threads can access GPU caches at the same time. For instance, on the Nvidia Pascal P100, 2K and 112K threads can simultaneously access the same $24 \mathrm{~KB} \mathrm{~L} 1$ and $4 \mathrm{MB} \mathrm{L} 2$ caches. If each thread accesses unique memory locations, then each thread can only use $\frac{24 K}{2 K}=12 B /$ threads $\mathrm{L} 1$ and $\frac{4 M B}{112 K}=37 B /$ threads L2 cache. However, GPUs have a large number of registers and shared memory per Streaming Multiprocessor (SM). For example, $\mathrm{P} 100$ has $256 \mathrm{~KB}$ storage capacity in registers and $64 \mathrm{~KB}$ shared memory per each SM. The shared memory bandwidth on the P100 is higher than L1 cache. Therefore, utilizing these resources for improving locality would be beneficial for performance. In GPUs, only the memory locations with statically resolvable access patterns can be placed in registers. Since we process each row sequentially, the access pattern of $O$ can be statically determined, and these elements can be placed in registers. However, the accesses for $D$ depend on the sparsity structure; hence the accesses are kept in shared memory.

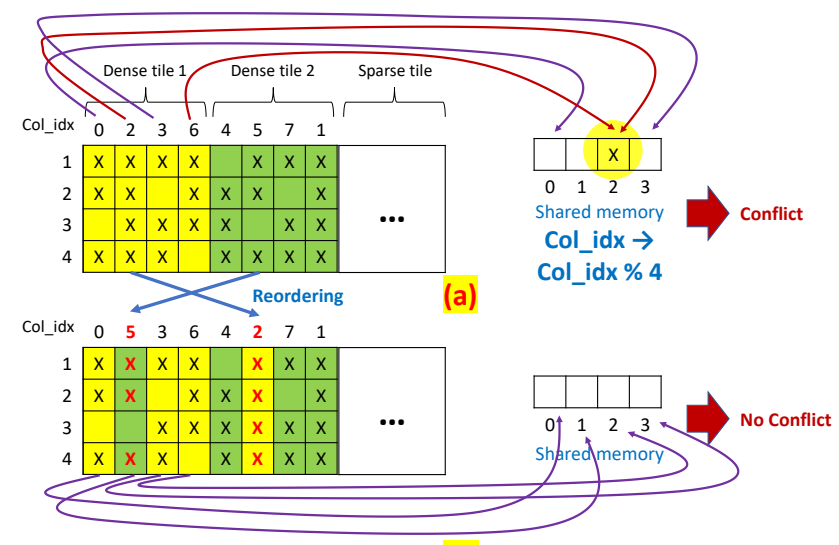

(b)

Figure 8. Remove Index Mapping Conflicts to Shared Memory

We next describe the approach to mapping of columns of $D$ to shared memory. Consider Fig. 8 and assume that column 'i' of $D$ is mapped to column $i \% 4$-th column in the shared memory. This would result in mapping the first (col_idx:2) and third columns (col_idx:6) of the yellow tile to the same location in shared memory (Fig. 8 (a)) resulting in a conflict. Alternatively, the needed columns of D could be mapped contiguously in shared memory, with an indirection array to indicate the mapping of column indices to shared memory. However, this strategy incurs two major overheads: i) extra 
Listing 2. SpMM with ASpT on GPUs (dense tile)

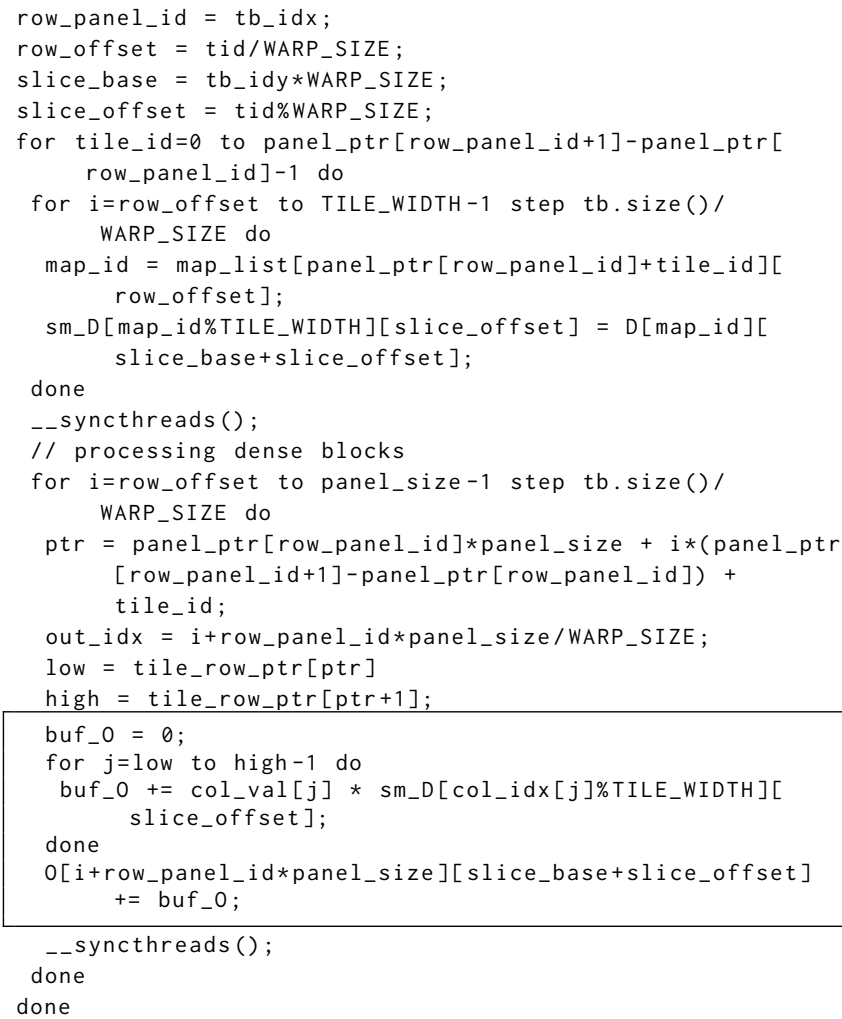

Listing 3. SpMM with ASpT on GPUs (sparse tile)

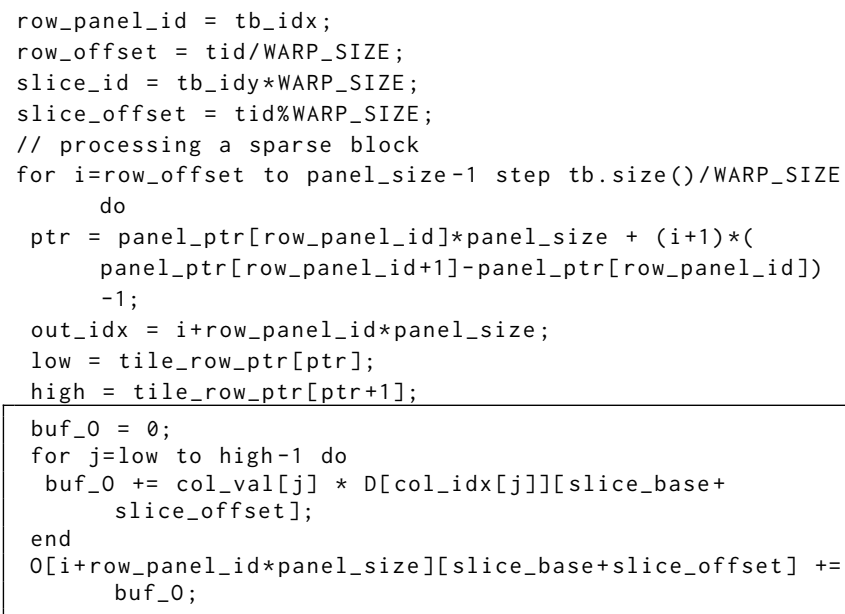

space required for the indirection array and ii) overhead due to access of the indirect array. For each non-zero access, the indirection array needs to be accessed to find the element in shared memory, which is inefficient. We addressed this issue by reordering column indices to remove mapping conflicts in each tile. That is, every column index in the tile is mapped to a different location in the shared memory. By doing so, we can directly access the shared memory using a simple modulo operation. For example, in Fig. 8 (b), 'modulo 4' mapping can be used. This strategy may result in partially filled tiles.
If a heavy 2D tile does not have enough column segments, they are moved to the sparse segment. The reordering can be easily done once during the pre-processing stage.

\subsubsection{SpMM Algorithm: GPUs}

Listing 2 and 3 show the SpMM-ASpT GPU algorithm for dense and sparse tiles, respectively. The GPU algorithm is similar to that for multi/many-cores. The different threads in a warp are mapped along $K$ to avoid thread divergence and to achieve good load balance. For heavy 2D tiles the corresponding elements of $D$ are brought to shared memory, whereas for light 2D tiles shared memory is not used. Each 2D tile is processed by a thread block.

For processing both dense and sparse tiles, row panel id, row offset, and slice index are first computed (line 1-4 in Listing 2 and 3). Then, for dense tiles, all the threads in a thread block collectively bring the corresponding elements of $D$ to shared memory (line 6-9 in Listing 2). map_id (line 7 in Listing 2) keeps track of the original column index, and is used to access elements of $D$.

The rest of the code (Lines 12-23 in Listing 2 and Lines 616 in Listing 3) is very similar to multi/many-core algorithm. Different warps process different rows within a row-panel, and the threads within a warp are distributed along $K$. The results are accumulated in registers and written out to globalmemory at the end of each row (Line 21 in Listing 2 and Line 15 in Listing 3).

\subsection{Parameter Selection}

The key parameters that affect performance for ASpT are i) the threshold for the number of non-zeros in a column segment to be classified as heavy and ii) the tile sizes $\left(T_{M}\right.$, $T_{N}$, and $T_{K}$ ). These parameters were empirically determined using synthetic matrices described in Sec. 3. Fig. 5 shows the performance of CSR, DCSC and 2D tile as a function of column density. The threshold for classifying a column segment as heavy is chosen as the minimum column density at which 2D tile outperforms CSR. The rationale is that heavy segments are processed by the $2 \mathrm{D}$ tile algorithm, whereas the light segment, even though represented as a single 2D tile, is processed by CSR algorithm. Thus, if the number of nonzeros in a column segment is less than the crossover point in Fig. 5, it is better to process those non-zeros using the CSR algorithm, and the 2D tile algorithm otherwise. The tile sizes were also chosen empirically such that the data footprint of the tile fits in the L2 cache (512 KB per core) for the KNL (i.e. $\left(T_{M}+T_{N}\right) \times T_{K} \times \operatorname{sizeof}($ word $) \times \frac{\# \_o f \_t h r e a d s}{\# \_ \text {_of cores }}$ $=512 \mathrm{~K})$. For our experiments, the $\mathrm{L} 1$ cache was too small to exploit locality (and thus did not give great benefits). We explored different $\left(T_{M}, T_{N}, T_{K}\right)$ subjected to the $\mathrm{L} 2$ footprint constraint, and selected the best performing parameters. The best performance was obtained when $T_{M}=T_{N}, T_{K}=K$, and $\frac{\text { \#_of_threads }}{\text { \#_of_cores }}=2$. 
We followed similar steps for selecting GPU parameters. Since $D$ elements are kept in shared memory, the tile sizes $T_{K}$ and $T_{N}$ are constrained by the shared memory capacity. The shared memory size per thread block was selected such that full occupancy was maintained (for P100 we assigned 32KB of Shared-Memory per thread block of size 1024). Since the elements of $O$ are kept in registers, $T_{K}$ and $T_{M}$ are constrained by the register capacity. Thread coarsening $[25,26]$ was also employed to improve performance.

\section{SDDMM with ASpT}

In SDDMM, two dense matrices are multiplied and the resulting matrix is then scaled using an element-wise multiplication (Hadamard product) with a sparse matrix. Since the sparsity structure of the input and output sparse matrices is the same, we can optimize SDDMM by forming dense matrix products only at locations corresponding to non-zero elements in the input sparse matrix, as done by existing implementations $[11,20]$.

Listing 4. Part of SDDMM on multi cores

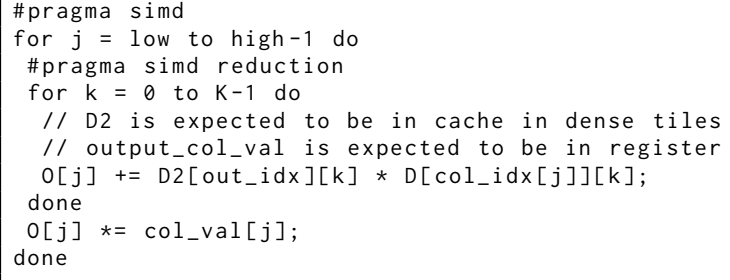

Listing 5. Part of SDDMM on GPUs (dense tile)

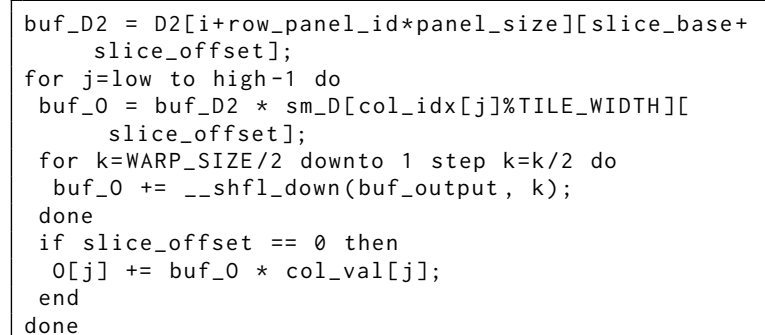

Listing 6. Part of SDDMM on GPUs (sparse tile)

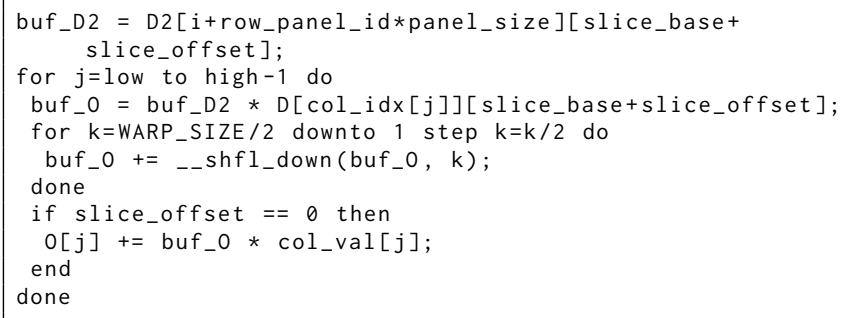

SDDMM on multi/many-cores can be implemented by substituting the box (line 11-18) in Listing 1 with Listing 4. For SDDMM, the $K$ dimension is not tiled, and the tile sizes $T_{M}$ and $T_{N}$ are chosen such that both D1 and D2 fit in L2 cache. The for loop in Line 11 computes the dot product of
D1 and D2. The inner for loop (Line 14) corresponding to $K$ is vectorized. Unlike SpMM, SDDMM requires reduction across the $\mathrm{K}$ dimension and is implemented by specifying the 'reduction clause' in Line 17. Line 19 scales the result by multiplying it with the corresponding element in the input sparse matrix $S$.

SDDMM on GPUs for dense and sparse tiles can be implemented by replacing the box in Listing 2 and 3 by Listing 5 and 6, respectively. The only difference between Listing 5 and 6 is in Line 13 where elements of $D 1$ are served by shared-memory in the dense version and global memory in the sparse version. The elements of D2 corresponding to the row are kept in registers for both dense and sparse tiles (line 11 in Listing 5). Since the $K$ dimension is mapped across threads and the corresponding $O$ elements are kept in registers that are private to a thread, we use warp shuffling for reduction (line 14-16 in Listing 5). The accumulated output value is scaled and written back to global memory (line 17-19 in Listing 5).

\section{Experimental Evaluation}

This section details the experimental evaluation of the ASpTbased SpMM and SDDMM on three different architectures:

- Nvidia P100 GPU (56 Pascal SMs, 16GB global memory with bandwidth of $732 \mathrm{~GB} / \mathrm{sec}, 4 \mathrm{MB}$ L2 cache, and $64 \mathrm{~KB}$ shared memory per each SM)

- Intel Xeon Phi (68 cores at $1.40 \mathrm{GHz}, 16 \mathrm{~GB}$ MCDRAM with bandwidth of $384 \mathrm{~GB} / \mathrm{sec}$, 34MB L2 cache)

- Intel Xeon CPU E5-2680 v4 $(2 \times 14$ cores at $2.40 \mathrm{GHz}$, 16GB MCDRAM with bandwidth of $72 \mathrm{~GB} / \mathrm{sec}, 35 \mathrm{MB}$ L3 cache)

For GPU experiments, the code was compiled using NVCC 9.1 with the -O3 flag and was run with ECC turned off.

For the Intel Xeon Phi, the code was compiled using Intel ICC 18.0.0 with -O3 and -MIC-AVX512 flags. The clustering mode was set to 'All-to-All' and the memory mode was set to 'cache-mode' (to fit big datasets).

For the Intel Xeon CPU, the code was also compiled with Intel ICC 18.0.0 with -O3 flag.

We only include the kernel execution time for all experiments. Preprocessing time and data transfer time from CPU to GPU or disk to RAM are not included. The impact of preprocessing overhead is reported separately. All tests were run five times, and average numbers are reported.

\subsection{Datasets and Comparison Baseline}

For experimental evaluation, we selected 975 matrices from the SuiteSparse collection [13], using all matrices with at least $10 \mathrm{~K}$ rows, $10 \mathrm{~K}$ columns, and $100 \mathrm{~K}$ non-zeros. The matrices in SuiteSparse are from diverse application domains and represent a wide range of sparsity patterns.

For SpMM on KNL, we compared ASpT with Intel MKL [43], CSB [2], and TACO [20], which represent the current state-of-the-art SpMM implementations. 


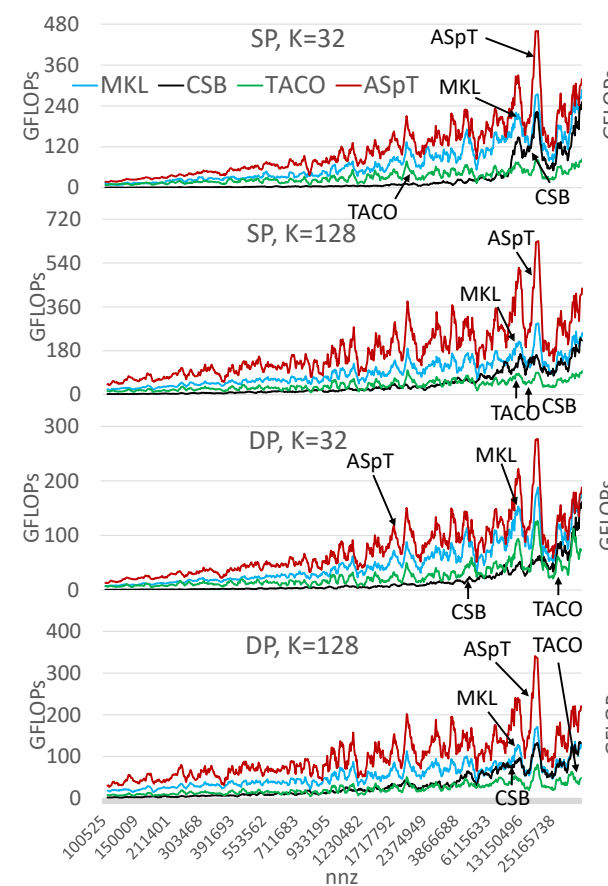

(a) SpMM results on KNL

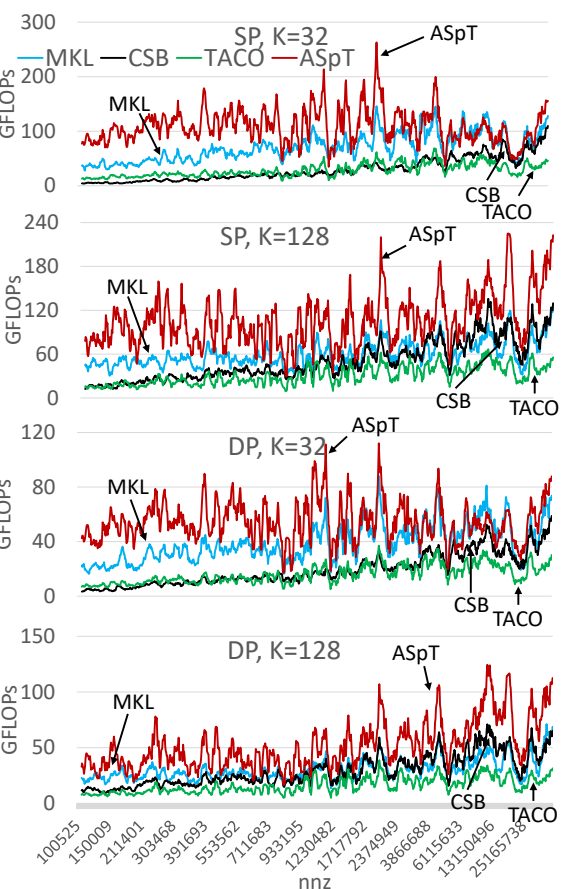

(b) SpMM results on Xeon

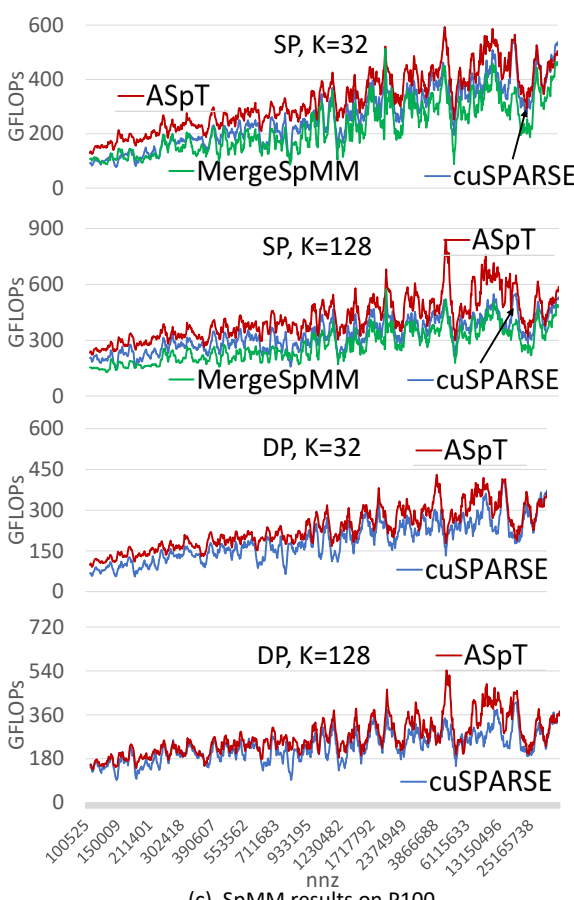

(c) SpMM results on $\mathrm{P} 100$

Figure 9. SpMM results

Table 1. Summary performance comparison: SpMM

\begin{tabular}{|c|c|c|c|c|c|c|c|c|c|c|c|c|c|}
\hline & & \multirow{2}{*}{\multicolumn{12}{|c|}{ percentage }} \\
\hline & & & & & & & & & & & & & \\
\hline & & \multicolumn{4}{|c|}{ KNL } & \multicolumn{4}{|c|}{ Xeon } & \multicolumn{4}{|c|}{ GPU } \\
\hline & & \multicolumn{2}{|c|}{ SP } & \multicolumn{2}{|c|}{ DP } & \multicolumn{2}{|c|}{ SP } & \multicolumn{2}{|c|}{ DP } & \multicolumn{2}{|c|}{ SP } & \multicolumn{2}{|c|}{ DP } \\
\hline & & $\mathrm{K}=32$ & $\mathrm{~K}=128$ & $\mathrm{~K}=32$ & $\mathrm{~K}=128$ & $\mathrm{~K}=32$ & $\mathrm{~K}=128$ & $\mathrm{~K}=32$ & $\mathrm{~K}=128$ & $\mathrm{~K}=32$ & $\mathrm{~K}=128$ & $\mathrm{~K}=32$ & $\mathrm{~K}=128$ \\
\hline \multirow{4}{*}{ slowdown } & $>100 \%$ & $1.7 \%$ & $1.4 \%$ & $1.4 \%$ & $1.7 \%$ & $5.1 \%$ & $1.0 \%$ & $4.0 \%$ & $1.2 \%$ & $0.1 \%$ & $0.0 \%$ & $0.1 \%$ & $0.1 \%$ \\
\hline & $50 \% \sim 100 \%$ & $1.9 \%$ & $0.7 \%$ & $1.2 \%$ & $1.4 \%$ & $6.7 \%$ & $3.6 \%$ & $7.9 \%$ & $2.6 \%$ & $0.1 \%$ & $0.0 \%$ & $0.1 \%$ & $0.4 \%$ \\
\hline & $10 \% \sim 50 \%$ & $4.2 \%$ & $4.0 \%$ & $5.2 \%$ & $6.1 \%$ & $12.6 \%$ & $13.9 \%$ & $16.4 \%$ & $10.8 \%$ & $1.7 \%$ & $1.7 \%$ & $3.2 \%$ & $10.4 \%$ \\
\hline & $0 \% \sim 10 \%$ & $3.2 \%$ & $2.5 \%$ & $4.1 \%$ & $3.3 \%$ & $6.2 \%$ & $7.3 \%$ & $6.3 \%$ & $5.9 \%$ & $11.2 \%$ & $6.2 \%$ & $12.3 \%$ & $18.8 \%$ \\
\hline \multirow{4}{*}{ speedup } & $0 \% \sim 10 \%$ & $2.6 \%$ & $3.0 \%$ & $3.7 \%$ & $3.9 \%$ & $4.2 \%$ & $7.4 \%$ & $7.4 \%$ & $6.9 \%$ & $24.9 \%$ & $18.8 \%$ & $21.4 \%$ & $22.9 \%$ \\
\hline & $10 \% \sim 50 \%$ & $19.4 \%$ & $15.8 \%$ & $25.0 \%$ & $20.2 \%$ & $15.7 \%$ & $26.7 \%$ & $20.3 \%$ & $32.2 \%$ & $49.5 \%$ & $53.5 \%$ & $44.1 \%$ & $34.0 \%$ \\
\hline & $50 \% \sim 100 \%$ & $35.0 \%$ & $28.8 \%$ & $33.1 \%$ & $32.5 \%$ & $18.5 \%$ & $20.6 \%$ & $15.3 \%$ & $24.6 \%$ & $6.1 \%$ & $16.4 \%$ & $5.1 \%$ & $3.6 \%$ \\
\hline & $>100 \%$ & $32.0 \%$ & $43.8 \%$ & $26.5 \%$ & $31.0 \%$ & $31.1 \%$ & $19.5 \%$ & $22.4 \%$ & $15.9 \%$ & $6.5 \%$ & $3.5 \%$ & $13.5 \%$ & $9.8 \%$ \\
\hline
\end{tabular}

For SpMM on GPUs, we compare ASpT with Nvidia cuSPARSE. cuSPARSE offers two modes, and we compare against the better performing one. We do not compare ASpT with MAGMA [3] and CUSP [12] as they are consistently outperformed by cuSPARSE (more than $40 \%$ on average).

For SDDMM on manycores (KNL), we compared ASpT with TACO which has been shown to significantly outperform Eigen [15] and uBLAS [42]. For SDDMM on GPUs, we compared ASpT with BIDMach [11], which represents the state-of-the-art SDMMM implementation.

We evaluate ASpT with single precision (SP) and double precision (DP), with the number of vectors set to 32 and
$128(\mathrm{~K}=32,128)$. However, only SP is used for SDDMM comparison on GPUs since BIDMach does not support DP for SDDMM.

\subsection{SpMM}

Fig. 9 (a) shows SpMM performance with SP and DP for different K widths on the KNL. Each point in Fig. 9 (a) represents the average GFLOPs value for a contiguous set of 10 matrices - the matrices are sorted in ascending order by the number of non-zeros. As shown in Fig. 9 (a), TACO is outperformed by CSB, which is outperformed by MKL. For all configurations, ASpT outperforms other implementations. ASpT performance is improved when $\mathrm{K}$ is increased from 


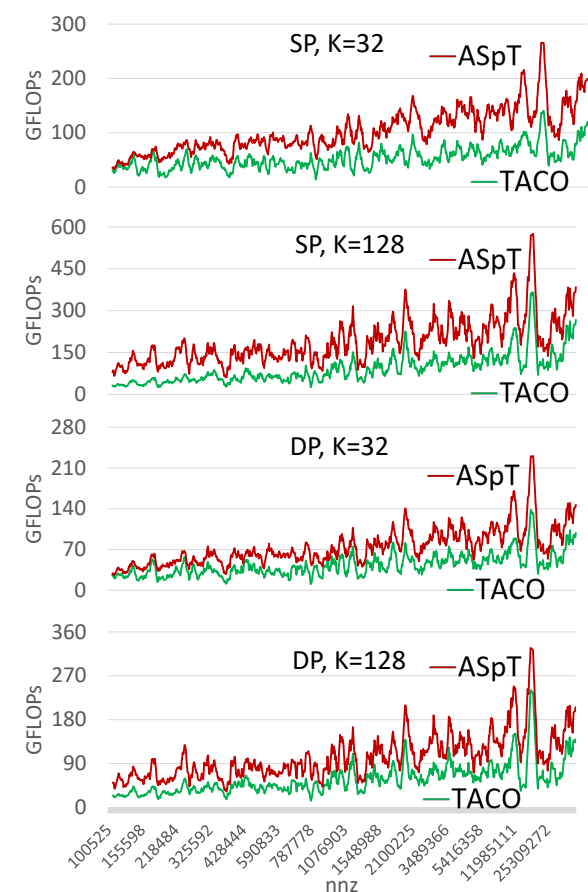

(a) SDDMM results on KNL

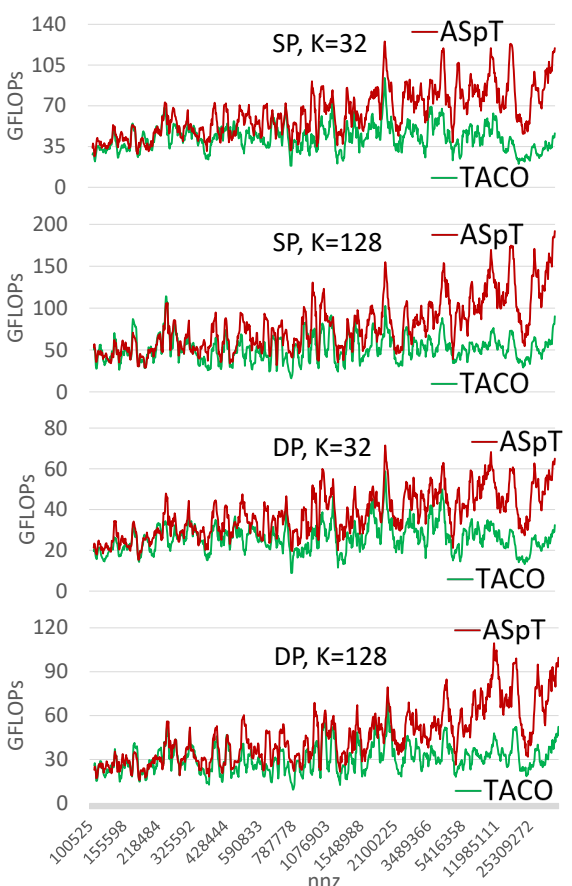

(b) SDDMM results on Xeon
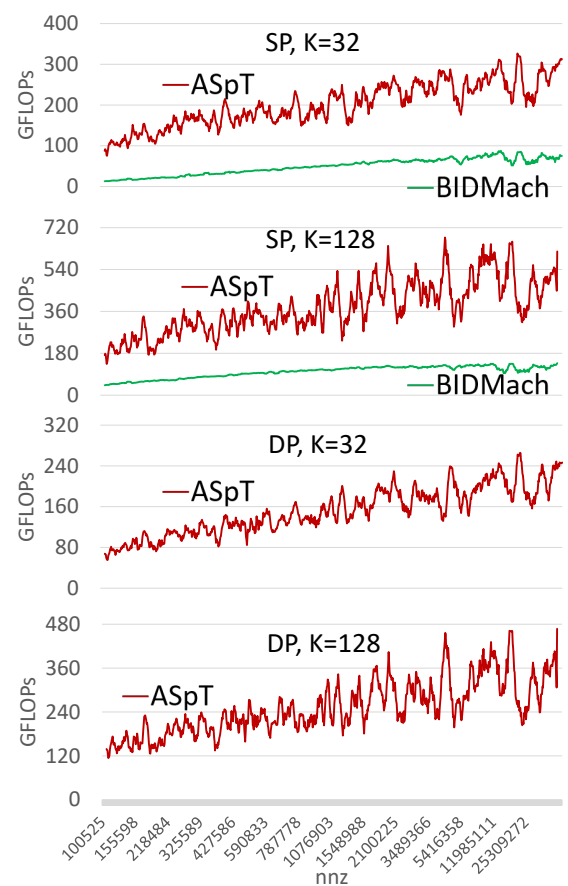

(c) SDDMM results on $\mathrm{P} 100$

Figure 10. SDDMM results

Table 2. Summary performance comparison: SDDMM

\begin{tabular}{|c|c|c|c|c|c|c|c|c|c|c|c|}
\hline & & \multirow{2}{*}{\multicolumn{10}{|c|}{ percentage }} \\
\hline & & & & & & & & & & & \\
\hline & & \multicolumn{4}{|c|}{ KNL } & \multicolumn{4}{|c|}{ Xeon } & \multirow{2}{*}{\multicolumn{2}{|c|}{$\begin{array}{c}\text { GPU } \\
\mathrm{SP}\end{array}$}} \\
\hline & & \multicolumn{2}{|c|}{ SP } & \multicolumn{2}{|c|}{ DP } & \multicolumn{2}{|c|}{ SP } & \multicolumn{2}{|c|}{ DP } & & \\
\hline & & $\mathrm{K}=32$ & $\mathrm{~K}=128$ & $\mathrm{~K}=32$ & $\mathrm{~K}=128$ & $\mathrm{~K}=32$ & $\mathrm{~K}=128$ & $\mathrm{~K}=32$ & $\mathrm{~K}=128$ & $\mathrm{~K}=32$ & $\mathrm{~K}=128$ \\
\hline \multirow{4}{*}{ slowdown } & $>100 \%$ & $0.0 \%$ & $0.0 \%$ & $0.0 \%$ & $0.0 \%$ & $0.0 \%$ & $0.5 \%$ & $0.0 \%$ & $0.1 \%$ & $0.0 \%$ & $0.0 \%$ \\
\hline & $50 \% \sim 100 \%$ & $0.1 \%$ & $0.2 \%$ & $0.3 \%$ & $0.1 \%$ & $1.3 \%$ & $2.3 \%$ & $0.1 \%$ & $1.5 \%$ & $0.0 \%$ & $0.0 \%$ \\
\hline & $10 \% \sim 50 \%$ & $2.2 \%$ & $2.5 \%$ & $4.2 \%$ & $3.6 \%$ & $15.6 \%$ & $15.5 \%$ & $10.6 \%$ & $11.3 \%$ & $0.0 \%$ & $0.2 \%$ \\
\hline & $0 \% \sim 10 \%$ & $3.3 \%$ & $1.4 \%$ & $3.1 \%$ & $2.8 \%$ & $11.2 \%$ & $7.0 \%$ & $15.7 \%$ & $9.1 \%$ & $0.0 \%$ & $0.0 \%$ \\
\hline \multirow{4}{*}{ speedup } & $0 \% \sim 10 \%$ & $2.7 \%$ & $2.2 \%$ & $3.8 \%$ & $3.2 \%$ & $4.5 \%$ & $9.0 \%$ & $9.0 \%$ & $9.5 \%$ & $0.0 \%$ & $0.1 \%$ \\
\hline & $10 \% \sim 50 \%$ & $16.3 \%$ & $14.4 \%$ & $18.6 \%$ & $22.8 \%$ & $20.5 \%$ & $17.8 \%$ & $20.4 \%$ & $25.4 \%$ & $0.2 \%$ & $1.2 \%$ \\
\hline & $50 \% \sim 100 \%$ & $21.8 \%$ & $24.9 \%$ & $27.5 \%$ & $29.3 \%$ & $14.1 \%$ & $16.3 \%$ & $19.8 \%$ & $17.8 \%$ & $1.4 \%$ & $11.3 \%$ \\
\hline & $>100 \%$ & $53.6 \%$ & $54.4 \%$ & $42.5 \%$ & $38.2 \%$ & $32.8 \%$ & $31.6 \%$ & $24.5 \%$ & $25.3 \%$ & $98.4 \%$ & $87.2 \%$ \\
\hline
\end{tabular}

32 to 128 . For matrices having a small number of non-zeros, performance is low. This is because concurrency is very low or there is not enough data reuse (i.e., $\frac{n n z}{\# \_o f \_c o l s}$ is small). An overall summary of the relative performance across the set of matrices is presented in Table 1 . The speedup and slowdown are defined as $\frac{\text { GFLOPs_with_ASPT }}{\text { GFLOPs_with_best_comparsion_baseline }}-1$ and $\frac{\text { GFLOPs_with_best_comparsion_baseline }}{\text { GFLOPs_with_ASpT }}-1$, respectively. ASpT achieves significant speedup for most matrices and only suffers slowdown for a small fraction of the matrices.

Fig. 9 (b) shows SpMM performance with SP and DP for different $\mathrm{K}$ widths on the Intel Xeon multicore CPU. As shown in Fig. 9, the relative performance trends of Xeon and KNL are quite similar.
Fig. 9 (c) shows SpMM performance on the Nvidia Pascal P100 GPU. The performance gap between ASpT and cuSPARSE is higher for higher $\mathrm{K}$ widths. The performance of cuSPARSE does not improve much when $\mathrm{K}$ is increased. cuSPARSE at most achieves 685 GFLOPs (SP, K=128 with tsyl201 dataset), whereas ASpT achieves around 900 GFLOPs when the number of non-zeros is around 4M. Fig. 9 (c) also compares ASpT with Merge-SpMM [48]. The comparison was limited to single precision as Merge-SpMM does not support double precision. For each dataset, Merge-SpMM reports GFLOPs with different strategies, and we took the best among them. Merge-SpMM's performance is slightly inferior to cuSPARSE, which is outperformed by ASpT. 


\subsection{SDDMM}

Figs. 10 (a) and (b) show the SDDMM performance on the KNL and Xeon, respectively. The performance trend is similar to SpMM in Figs. 9 (a) and (b), but the absolute GFLOPs is lower. On KNL, ASpT outperforms TACO for the majority of the datasets as shown in Table 2. Table 2 also shows a similar trend on Xeon CPU.

Fig. 10 (c) presents SDDMM performance on GPUs. The performance trend is similar to that of SpMM in Fig. 9 (c), with lower absolute GFLOPs. Both BIDMach and ASpT improve when $\mathrm{K}$ is increased, and ASpT significantly outperforms BIDMach across all the matrices. We only report BIDMach performance for single precision since it does not support double precision.

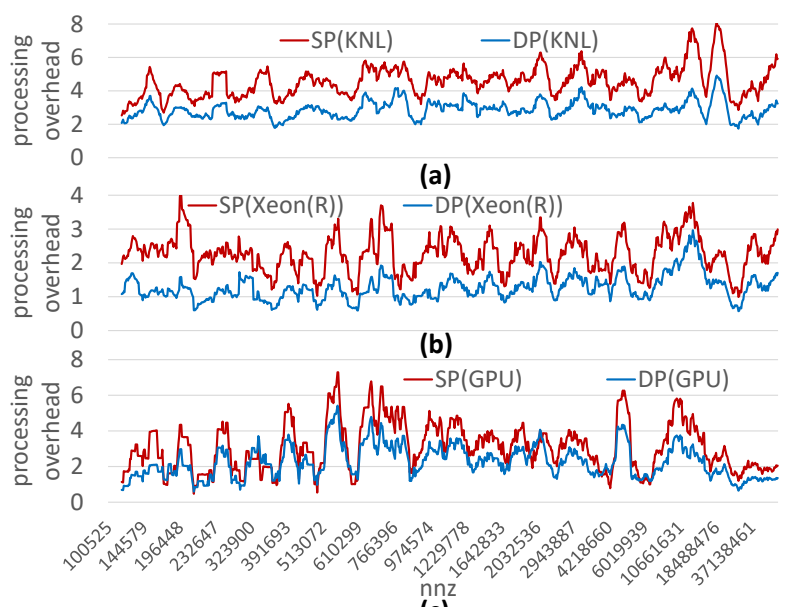

(c)

Figure 11. Preprocessing time (SpMM)

Table 3. Details of preprocessing overhead

\begin{tabular}{|c|c|c|c|c|c|c|}
\hline $\begin{array}{c}\text { ratio } \\
\text { (preprocessing_time } \\
\text { /computing_time) }\end{array}$ & $\begin{array}{c}\text { KNL } \\
(\mathrm{SP})\end{array}$ & $\begin{array}{c}\text { KNL } \\
\text { (DP) }\end{array}$ & $\begin{array}{c}\text { CPU } \\
\text { (SP) }\end{array}$ & $\begin{array}{c}\text { CPU } \\
(\mathrm{DP})\end{array}$ & $\begin{array}{c}\text { GPU } \\
(\mathrm{SP})\end{array}$ & $\begin{array}{c}\text { GPU } \\
(\mathrm{DP})\end{array}$ \\
\hline $0 \sim 5$ & $64.7 \%$ & $89.7 \%$ & $87.8 \%$ & $98.8 \%$ & $75.4 \%$ & $83.7 \%$ \\
\hline $5 \sim 10$ & $31.1 \%$ & $9.4 \%$ & $11.3 \%$ & $1.0 \%$ & $14.5 \%$ & $13.6 \%$ \\
\hline $10 \sim 15$ & $3.2 \%$ & $0.6 \%$ & $0.9 \%$ & $0.1 \%$ & $7.2 \%$ & $2.2 \%$ \\
\hline $15 \sim 20$ & $0.8 \%$ & $0.2 \%$ & $0.0 \%$ & $0.0 \%$ & $2.0 \%$ & $0.4 \%$ \\
\hline $20 \sim 25$ & $0.0 \%$ & $0.0 \%$ & $0.0 \%$ & $0.0 \%$ & $0.6 \%$ & $0.1 \%$ \\
\hline $25 \sim 30$ & $0.1 \%$ & $0.0 \%$ & $0.0 \%$ & $0.0 \%$ & $0.2 \%$ & $0.0 \%$ \\
\hline
\end{tabular}

\subsection{Preprocessing Overhead}

Constructing additional meta-data and reordering the column indices incurs overhead. Fig. 11 shows the preprocessing time normalized to the execution time of one ASpT SpMM with $\mathrm{K}=128$, for single precision (red curve) and double precision (blue curve). Typical applications involving SpMM and SDDMM execute a large number of iterations (e.g., [31] for SpMM and [51] for SDDMM). Hence, our preprocessing overhead is negligible. DP precision has less overhead, as the preprocessing time for SP and DP is similar but the SpMM time for DP is higher than that of SP. Table 3 shows that the preprocessing times are generally between $0-5 \times$ compute time.

Table 4. Benefit of tiling or reordering

\begin{tabular}{|c|c|c|}
\hline & Tiling-Only & Tiling+Reordering \\
\hline SpMM(KNL)/speed-up over MKL & 1.38 & 2.06 \\
\hline SpMM(GPU)/speed-up over cuSPARSE & 0.8 & 1.34 \\
\hline SDDMM(KNL)/speed-up over TACO & 1.02 & 2.01 \\
\hline SDDMM(GPU)/speed-up over BIDMach & 2.41 & 4.04 \\
\hline
\end{tabular}

\subsection{Benefit from Tiling / Reordering}

While tiling helps to improve the data-reuse of the dense input matrix, it has two main disadvantages (1) tiling overhead and (2) loss of concurrency for very sparse tiles. Tiling only helps to improve the data-reuse for columns with sufficient density. Hence, tiling overheads can be minimized by limiting the tiling to columns with sufficient column density. A simple 2D tiling strategy would include columns of both low and high density which may affect performance. The performance can be improved by grouping columns with high density and limiting tiling to these high-density columns; This can be achieved using reordering. Note that reordering without tiling may not improve performance as the datareuse may not be improved. However, it is possible that the inherent matrix structure may already be clustered and thus tiling without any reordering could potentially improve performance. Hence, we ran experiments with just tiling and no reordering. Results with $\mathrm{K}=128$ for single precision are presented in Table 4; other configurations achieved similar results. As shown in Table 4, the combined Tiling+Reordering strategy substantially outperforms the Tiling-Only strategy.

\section{Conclusion}

SpMM and SDDMM are key kernels in many machine learning applications. In contrast to other efforts that use customized sparse matrix representations to achieve high performance, this paper targets efficient implementation of these primitives using the standard (unordered) CSR sparse matrix representation so that incorporation into applications can be facilitated. An adaptive 2D tiled approach exposes higher memory reuse potential, and an efficient reordering scheme enables efficient execution of $2 \mathrm{D}$ tiles. In comparison to the current state-of-the-art, the ASpT based SpMM algorithm achieves a speedup of up to $13.18 \mathrm{x}$, with a geometric mean of $1.65 \mathrm{x}$ on an Intel Xeon Phi KNL; a speedup of up to $7.26 \mathrm{x}$, with a geometric mean of $1.36 \mathrm{x}$ on an Intel Xeon multicore processor; and a speedup of up to $24.21 \mathrm{x}$ with a geometric mean of $1.35 \mathrm{x}$ on GPUs. The ASpT based SDDMM algorithm achieves a speedup of up to $30.15 \mathrm{x}$, with a geometric mean of $1.93 \mathrm{x}$ on the KNL; a speed of up to $22.75 \mathrm{x}$, with a geometric 
mean of $1.52 \mathrm{x}$ on the Xeon; and a speedup of up to $13.74 \mathrm{x}$, with a geometric mean of 3.60x on GPUs.

\section{Acknowledgments}

We thank the reviewers for the valuable feedback and the Ohio Supercomputer Center for use of their resources. This work was supported in part by the Defense Advanced Research Projects Agency under Contract D16PC00183, and the National Science Foundation (NSF) through awards 1404995, 1513120, 1629548, 1645599, and 1816793.

\section{A Artifact Appendix}

\section{A.1 Abstract}

The artifact contains the implementation of sparse-matrix dense-matrix multipilicationo (SpMM) and sampled densedense matrix multiplication (SDDMM) described in PPoPP 2019 paper titled "Adaptive Sparse Tiling for Sparse Matrix Multiplication". In addition to the source code, associated scripts to replicate the experimental evaluation are provided. The "compile*.sh" script will automatically install the code. The required datasets can be downloaded using the "download.sh". The results can be validated by running the "run*.sh" script.

\section{A.2 Description}

\section{A.2.1 Check-list (Artifact Meta Information)}

- Required Compilers:

- nvcc 9.1.85

- gcc 4.8 .5 or 4.9 .3

- icc 18.0.3 (with MKL)

- Data set:

- All datasets were downloaded from SuiteSparse (http://faculty.cse.tamu.edu/davis/suitesparse.html)

- A script called "download.sh" will automatically download all the required datasets. This include the datasets which were used to evaluate the performance of implementations.

- Run-time environment:

- OS: Linux/Windows (the results presented in the paper are based on experiments run on a Linux platform)

- Root access: Required to turn off ECC on GPU and set the clustering mode to 'All-to-All' and the memory mode to 'cache-mode' on KNL (both are not required for functional verification)

\section{- Hardware: manycore}

- For performance verification: Intel Xeon Phi (68 cores with $1.40 \mathrm{GHz}, 16 \mathrm{~GB}$ MCDRAM with bandwidth of $384 \mathrm{~GB} / \mathrm{sec}$, 34MB L2 cache)

- For functional verification: AVX-512

- Hardware: multicore

- For performance verification: Intel Xeon CPU E5-2680 v4 $(2 \times 14$ cores with $2.40 \mathrm{GHz}, 16 \mathrm{~GB}$ MCDRAM with bandwidth of $72 \mathrm{~GB} / \mathrm{sec}, 35 \mathrm{MB}$ L3 cache)

- Hardware: GPU

- For performance verification: Nvidia Pascal P100
- For functional verification: Any Nvidia GPU with compute capability $\geq 6.0$ and global memory $\geq 16 \mathrm{~GB}$

- Execution:

- For performance verification: sole user

- Output:

- Console

- Experiment replication:

- Bash script is provided with the distribution

- External dependences:

- cmake $>=3.11 .4$ (Not present in distribution)

- boost $>=1.58$ (Not present in distribution)

- Apache Maven 3.6.0 (Not present in distribution)

- JDK 8 (Not present in distribution)

- Publicly available?:

- Yes. Link: http://gitlab.hpcrl.cse.ohio-state.edu/chong/ppopp19_ae

\section{A.2.2 How Delivered}

The source code of ASpT, associated scripts to install/run/verify the framework and scripts to download datasets are available at http://gitlab.hpcrl.cse.ohio-state.edu/chong/ppopp19_ae

\section{A.3 Installation}

After cloning/downloading the source code, follow these steps.

- run "compile*.sh”. For example, “./compile_GPU_SpMM.sh" will install all GPU SpMM implementations

- run "download.sh". This script will download all the datasets. The results section of the paper contain results with the dataset

\section{A.4 Experiment Workflow}

Ensure that implementations and associated dependences are installed properly. Download the datasets using the "download.sh" script. To run the benchmarks use the "run*.sh" script. For eg. running “./run_GPU_SpMM.sh” will run all GPU SpMM implementations.

\section{A.5 Evaluation and Expected Result}

The expected results are shown in Figs. 9 through 11 and Table 1 and 2 . The performance may vary depending on the machines used. Functional correctness can be evaluated on any Nvidia GPU with compute capability $\geq$ than 6.0 and a manycore processor with AVX-512.

\section{References}

[1] 2018. The API reference guide for cuSPARSE, the CUDA sparse matrixlibrary.(v9.1 ed.). http://docs.nvidia.com/cuda/cusparse/index. html.

[2] Hasan Metin Aktulga, Aydin Buluç, Samuel Williams, and Chao Yang. 2014. Optimizing sparse matrix-multiple vectors multiplication for nuclear configuration interaction calculations. In Parallel and Distributed Processing Symposium, 2014 IEEE 28th International. IEEE, 1213-1222.

[3] Hartwig Anzt, Stanimire Tomov, and Jack Dongarra. 2015. Accelerating the LOBPCG method on GPUs using a blocked sparse matrix vector product. In Proceedings of the Symposium on High Performance Computing. Society for Computer Simulation International, 75-82. 
[4] Allison H Baker, John M Dennis, and Elizabeth R Jessup. 2006. On improving linear solver performance: A block variant of GMRES. SIAM Journal on Scientific Computing 27, 5 (2006), 1608-1626.

[5] Nathan Bell and Michael Garland. 2009. Implementing sparse matrixvector multiplication on throughput-oriented processors. In Proceedings of the conference on high performance computing networking, storage and analysis. ACM, 18.

[6] Aydin Buluc and John R Gilbert. 2008. On the representation and multiplication of hypersparse matrices. In Parallel and Distributed Processing, 2008. IPDPS 2008. IEEE International Symposium on. IEEE, 1-11.

[7] Aydin Buluc, Tim Mattson, Scott McMillan, José Moreira, and Carl Yang. 2017. Design of the GraphBLAS API for C. In Parallel and Distributed Processing Symposium Workshops (IPDPSW), 2017 IEEE International. IEEE, 643-652.

[8] Aydin Buluc, Samuel Williams, Leonid Oliker, and James Demmel. 2011. Reduced-bandwidth multithreaded algorithms for sparse matrixvector multiplication. In Parallel \& distributed processing symposium (IPDPS), 2011 IEEE international. IEEE, 721-733.

[9] Alfredo Buttari, Victor Eijkhout, Julien Langou, and Salvatore Filippone. 2007. Performance optimization and modeling of blocked sparse kernels. The International fournal of High Performance Computing Applications 21, 4 (2007), 467-484.

[10] John Canny. 2002. Collaborative filtering with privacy. In Security and Privacy, 2002. Proceedings. 2002 IEEE Symposium on. IEEE, 45-57.

[11] John Canny and Huasha Zhao. 2013. Bidmach: Large-scale learning with zero memory allocation. In BigLearn workshop, NIPS. 117.

[12] Steven Dalton, Nathan Bell, Luke Olson, and Michael Garland. 2014. Cusp: Generic parallel algorithms for sparse matrix and graph computations, 2014. Version 0.5. 0 (2014).

[13] Timothy A Davis and Yifan Hu. 2011. The University of Florida sparse matrix collection. ACM Transactions on Mathematical Software (TOMS) 38, 1 (2011), 1.

[14] Joseph L Greathouse and Mayank Daga. 2014. Efficient sparse matrixvector multiplication on GPUs using the CSR storage format. In Proceedings of the International Conference for High Performance Computing, Networking, Storage and Analysis. IEEE Press, 769-780.

[15] Gaël Guennebaud, Benort Jacob, Philip Avery, Abraham Bachrach, Sebastien Barthelemy, et al. 2010. Eigen v3.

[16] Song Han, Huizi Mao, and William J Dally. 2015. Deep compression: Compressing deep neural networks with pruning, trained quantization and huffman coding. arXiv preprint arXiv:1510.00149 (2015).

[17] Changwan Hong, Aravind Sukumaran-Rajam, Bortik Bandyopadhyay, Jinsung Kim, Süreyya Emre Kurt, Israt Nisa, Shivani Sabhlok, Ümit V. Çatalyürek, Srinivasan Parthasarathy, and P. Sadayappan. 2018. Efficient Sparse-matrix Multi-vector Product on GPUs. In Proceedings of the 27th International Symposium on High-Performance Parallel and Distributed Computing (HPDC '18). ACM, New York, NY, USA, 66-79. https://doi.org/10.1145/3208040.3208062

[18] George Karypis and Vipin Kumar. 1998. A fast and high quality multilevel scheme for partitioning irregular graphs. SIAM fournal on scientific Computing 20, 1 (1998), 359-392.

[19] Christoph W Keßler and Craig H Smith. 1999. The SPARAMAT approach to automatic comprehension of sparse matrix computations. In Program Comprehension, 1999. Proceedings. Seventh International Workshop on. IEEE, 200-207.

[20] Fredrik Kjolstad, Shoaib Kamil, Stephen Chou, David Lugato, and Saman Amarasinghe. 2017. The tensor algebra compiler. Proceedings of the ACM on Programming Languages 1, OOPSLA (2017), 77.

[21] Yehuda Koren, Robert Bell, and Chris Volinsky. 2009. Matrix factorization techniques for recommender systems. Computer 8 (2009), 30-37.

[22] Kartik Lakhotia, Shreyas Singapura, Rajgopal Kannan, and Viktor Prasanna. 2017. ReCALL: Reordered Cache Aware Locality based Graph Processing. In High Performance Computing (HiPC), 2017 IEEE 24th International Conference on. IEEE, 273-282.
[23] Weifeng Liu and Brian Vinter. 2015. CSR5: An efficient storage format for cross-platform sparse matrix-vector multiplication. In Proceedings of the 29th ACM on International Conference on Supercomputing. ACM, 339-350.

[24] Yongchao Liu and Bertil Schmidt. 2015. LightSpMV: Faster CSRbased sparse matrix-vector multiplication on CUDA-enabled GPUs. In Application-specific Systems, Architectures and Processors (ASAP), 2015 IEEE 26th International Conference on. IEEE, 82-89.

[25] Alberto Magni, Christophe Dubach, and Michael O'Boyle. 2014. Automatic optimization of thread-coarsening for graphics processors. In Proceedings of the 23rd international conference on Parallel architectures and compilation. ACM, 455-466.

[26] Alberto Magni, Christophe Dubach, and Michael FP O’Boyle. 2013. A large-scale cross-architecture evaluation of thread-coarsening. In Proceedings of the International Conference on High Performance Computing, Networking, Storage and Analysis. ACM, 11.

[27] Duane Merrill and Michael Garland. 2016. Merge-based sparse matrixvector multiplication (SpMV) using the CSR storage format. In Proceedings of the 21st ACM SIGPLAN Symposium on Principles and Practice of Parallel Programming (PPoPP '16). ACM, New York, NY, USA, Article 43, 2 pages. https://doi.org/10.1145/2851141.2851190

[28] Alexander Monakov, Anton Lokhmotov, and Arutyun Avetisyan. 2010. Automatically Tuning Sparse Matrix-Vector Multiplication for GPU Architectures. HiPEAC 5952 (2010), 111-125.

[29] Leonid Oliker, Xiaoye Li, Parry Husbands, and Rupak Biswas. 2002. Effects of ordering strategies and programming paradigms on sparse matrix computations. Siam Review 44, 3 (2002), 373-393.

[30] Gloria Ortega, Francisco Vázquez, Inmaculada García, and Ester M Garzón. 2013. Fastspmm: An efficient library for sparse matrix matrix product on GPUs. Comput. J. 57, 7 (2013), 968-979.

[31] Jongsoo Park, Sheng Li, Wei Wen, Ping Tak Peter Tang, Hai Li, Yiran Chen, and Pradeep Dubey. 2016. Faster cnns with direct sparse convolutions and guided pruning. arXiv preprint arXiv:1608.01409 (2016).

[32] Juan C Pichel, Francisco F Rivera, Marcos Fernández, and Aurelio Rodríguez. 2012. Optimization of sparse matrix-vector multiplication using reordering techniques on GPUs. Microprocessors and Microsystems 36, 2 (2012), 65-77.

[33] Ahmet Erdem Sarıyüce, Erik Saule, Kamer Kaya, and Ümit V Çatalyürek. 2015. Regularizing graph centrality computations. f. Parallel and Distrib. Comput. 76 (2015), 106-119.

[34] Markus Steinberger, Andreas Derlery, Rhaleb Zayer, and Hans-Peter Seidel. 2016. How naive is naive SpMV on the GPU?. In High Performance Extreme Computing Conference (HPEC), 2016 IEEE. IEEE, 1-8.

[35] Markus Steinberger, Rhaleb Zayer, and Hans-Peter Seidel. 2017. Globally homogeneous, locally adaptive sparse matrix-vector multiplication on the GPU. In Proceedings of the International Conference on Supercomputing. ACM, 13.

[36] Bor-Yiing Su and Kurt Keutzer. 2012. clSpMV: A cross-platform OpenCL SpMV framework on GPUs. In Proceedings of the 26th ACM international conference on Supercomputing. ACM, 353-364.

[37] Xiangzheng Sun, Yunquan Zhang, Ting Wang, Xianyi Zhang, Liang Yuan, and Li Rao. 2011. Optimizing SpMV for diagonal sparse matrices on GPU. In Parallel Processing (ICPP), 2011 International Conference on. IEEE, 492-501.

[38] Wai Teng Tang, Wen Jun Tan, Rajarshi Ray, Yi Wen Wong, Weiguang Chen, Shyh-hao Kuo, Rick Siow Mong Goh, Stephen John Turner, and Weng-Fai Wong. 2013. Accelerating sparse matrix-vector multiplication on GPUs using bit-representation-optimized schemes. In Proceedings of the International Conference on High Performance Computing, Networking, Storage and Analysis. ACM, 26.

[39] Michalis K Titsias. 2008. The infinite gamma-Poisson feature model. In Advances in Neural Information Processing Systems. 1513-1520.

[40] Francisco Vázquez, José-Jesús Fernández, and Ester M Garzón. 2011. A new approach for sparse matrix vector product on NVIDIA GPUs. 
Concurrency and Computation: Practice and Experience 23, 8 (2011), 815-826.

[41] Richard Vuduc, James W Demmel, and Katherine A Yelick. 2005. OSKI: A library of automatically tuned sparse matrix kernels. In fournal of Physics: Conference Series, Vol. 16. IOP Publishing, 521.

[42] Joerg Walter, Mathias Koch, et al. 2006. uBLAS. Boost C++ software library available from http://www. boost. org/doc/libs (2006).

[43] Endong Wang, Qing Zhang, Bo Shen, Guangyong Zhang, Xiaowei Lu, Qing Wu, and Yajuan Wang. 2014. Intel math kernel library. In HighPerformance Computing on the Intel® Xeon PhiâĎć. Springer, 167-188.

[44] Hao Wei, Jeffrey Xu Yu, Can Lu, and Xuemin Lin. 2016. Speedup graph processing by graph ordering. In Proceedings of the 2016 International Conference on Management of Data. ACM, 1813-1828.

[45] Samuel Williams, Leonid Oliker, Richard Vuduc, John Shalf, Katherine Yelick, and James Demmel. 2007. Optimization of sparse matrix-vector multiplication on emerging multicore platforms. In Supercomputing, 2007. SC'07. Proceedings of the 2007 ACM/IEEE Conference on. IEEE, $1-12$.

[46] Biwei Xie, Jianfeng Zhan, Xu Liu, Wanling Gao, Zhen Jia, Xiwen He, and Lixin Zhang. 2018. CVR: efficient vectorization of SpMV on x86 processors. In Proceedings of the 2018 International Symposium on Code Generation and Optimization. ACM, 149-162.

[47] Shengen Yan, Chao Li, Yunquan Zhang, and Huiyang Zhou. 2014. yaSpMV: yet another SpMV framework on GPUs. In Acm Sigplan Notices, Vol. 49. ACM, 107-118.

[48] Carl Yang, Aydin Buluc, and John D Owens. 2018. Design Principles for Sparse Matrix Multiplication on the GPU. arXiv preprint arXiv:1803.08601 (2018).

[49] Hiroki Yoshizawa and Daisuke Takahashi. 2012. Automatic tuning of sparse matrix-vector multiplication for CRS format on GPUs. In Computational Science and Engineering (CSE), 2012 IEEE 15th International Conference on. IEEE, 130-136.

[50] AN Yzelman and Rob H Bisseling. 2009. Cache-oblivious sparse matrixvector multiplication by using sparse matrix partitioning methods. SIAM Journal on Scientific Computing 31, 4 (2009), 3128-3154.

[51] Huasha Zhao, Biye Jiang, John F Canny, and Bobby Jaros. 2015. Same but different: Fast and high quality gibbs parameter estimation. In Proceedings of the 21th ACM SIGKDD International Conference on Knowledge Discovery and Data Mining. ACM, 1495-1502. 